\title{
Rheumatoid polyarthritis suspected in an HIV patient with scleritis, peripheral ulcerative keratitis, and anterior uveitis
}

This article was published in the following Dove Press journal:

International Medical Case Reports Journal

4 February 2016

Number of times this article has been viewed

\author{
Christelle Domngang \\ Noche' \\ Madeleine Singwé-Ngandeu ${ }^{2}$ \\ Assumpta Lucienne Bella ${ }^{2}$ \\ 'Université des Montagnes, Yaoundé, \\ Cameroon; ${ }^{2}$ Université de Yaoundé I, \\ Yaoundé, Cameroon
}

Correspondence: Christelle Domngang Noche

Université des Montagnes,

PO Box 12 7I5 Yaoundé, Cameroon

Email dockrystlnoche@gmail.com
Introduction: Scleritis and peripheral ulcerative keratitis are ocular manifestations found in many inflammations and infections. Therefore, their association should prompt a search for inflammatory or infectious causes that may be life-threatening, especially in the context of AIDS due to HIV infection.

Findings: We report the case of a 37-year-old female, first seen in 2011 with a nodular scleritis in the right eye and a peripheral ulcerative keratitis, a necrotizing scleritis, and a granulomatous anterior uveitis in the left eye, in the context of chronic polyarthropathies that had evolved over 6 months. The patient was diagnosed with AIDS (HIV) in 2008 and was on antiretroviral therapy for the past 2 years. Ophthalmic workup was negative for opportunistic infections and potential causes of scleritis and peripheral ulcerative keratitis, and the patient was unresponsive to topical antibacterial and anti-inflammatory treatment. Ocular lesion resolution and articular swelling improvement was observed less than 6 weeks after sulfasalazine treatment. Based on American College of Rheumatology/European League Against Rheumatism classification criteria, and considering the good response to the treatment (sulfasalazine), diagnosis of rheumatoid arthritis was made in the absence of confirmatory lab tests results.

Conclusion: In the context of ocular manifestations associated with polyarthropathies, coexisting pathologies should be considered. Diagnostic workup of chronic inflammatory rheumatism should be carried out, even in the context of HIV/AIDS.

Keywords: scleritis, peripheral ulcerative keratitis, anterior uveitis, rheumatoid arthritis, HIV/ AIDS

\section{Introduction}

Many reports have recently described an association between chronic inflammatory rheumatism and HIV infection in Africa. ${ }^{1-10}$ More specifically, although the relationship between rheumatoid arthritis (RA) and HIV/AIDS infection, both life-threatening diseases, is possible, this association is rare. ${ }^{11}$ These diseases can affect different systems and can particularly lead to ocular involvement. Ocular lesions in RA and HIV/AIDS infection are well documented, ${ }^{12,13}$ although ocular manifestations in a combination of both diseases have not been described in the past years. We therefore report the case of a 37-year-old HIV-positive female, who exhibited an association of nodular scleritis, peripheral ulcerative keratitis, and anterior uveitis in the context of chronic polyarthropathies. To our knowledge, the coexistence of these types of ocular lesions in a similar context has not been reported previously. Written informed consent was obtained from the patient to publish this report and any accompanying images. Permission from the institutional review board of the Université des Montagnes was also obtained. 


\section{Findings}

A 37-year-old female presented to an eye unit in February 2011 with a 6-week history of significant ocular discomfort, associated with bilateral red eyes and a nodule in the right one.

Past medical history revealed significant polyarticular pain with joint swelling over the past 6 months (both shoulders, elbows, and wrists, interphalangeal joints of two fingers of the left hand, both knees, and both ankles). The patient, diagnosed as positive for HIV-1 group M in 2008, was on antiretroviral treatment and sulfonamides since 2009. At the moment of consultation, she was at clinical stage 2 of HIV/ AIDS (according to the World Health Organization).

Based on ocular examination, best-corrected visual acuities were 20/20 and 20/50 in the right and left eye, respectively. Slit-lamp examination of the right eye showed a superior nodular scleritis surrounded by mild inflammation, while in the left eye, a peripheral (temporoinferior) corneal ulceration associated with scleritis and granulomatous anterior uveitis (presence of cells and flare and absence of posterior synechiae in the anterior chamber) was observed (Figures 1 and 2). Intraocular pressure was $15 \mathrm{mmHg}$, and the fundus examination was normal in both eyes.

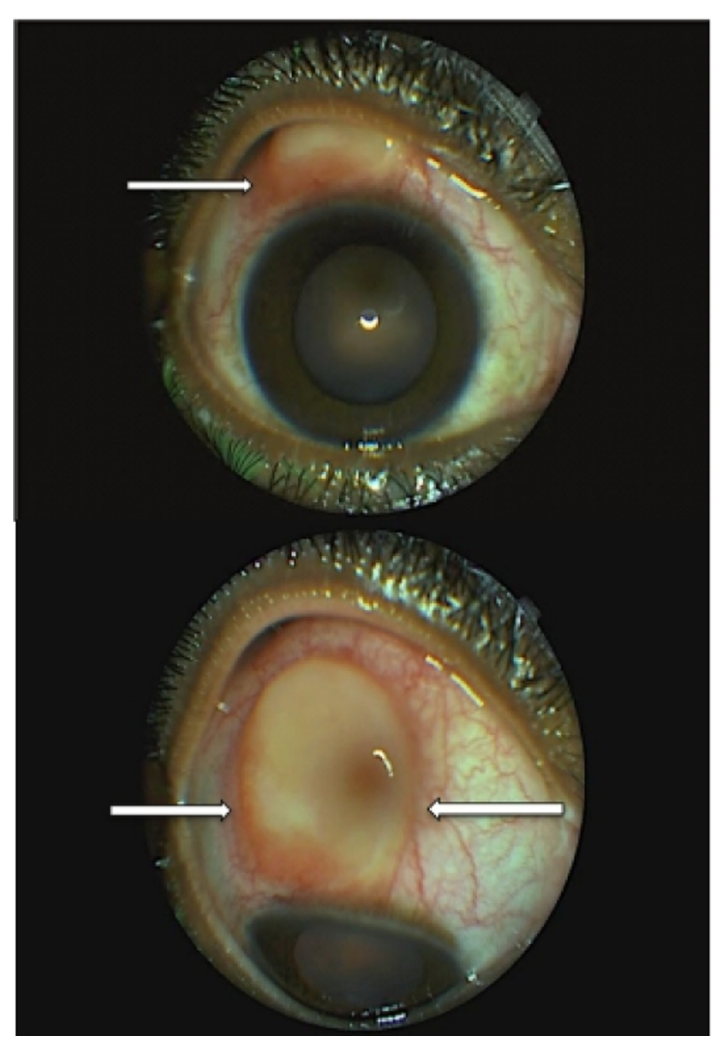

Figure I Right eye lesion before treatment with sulfasalazine.

Notes: White arrows show superior scleral node with mild perilesional inflammation and a quiet anterior chamber. Absence of epithelial defects (staining with fluorescein).
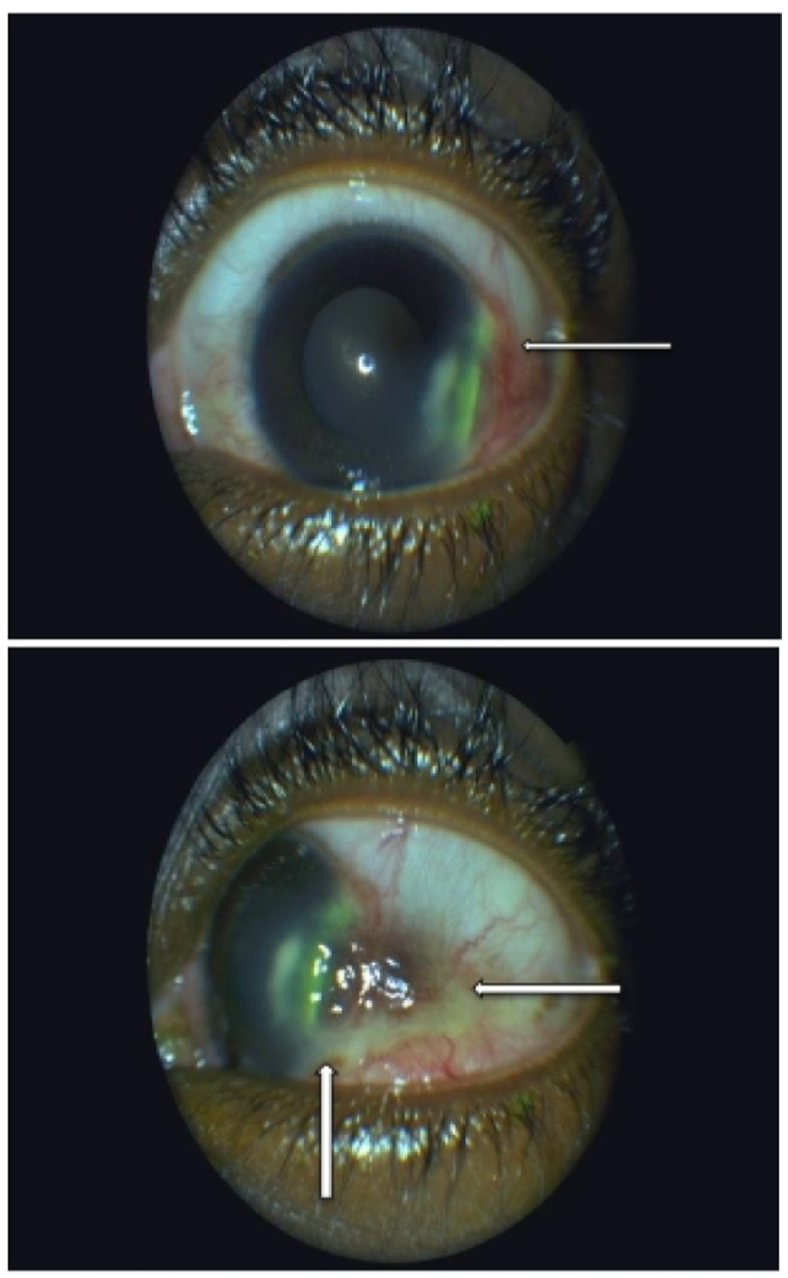

Figure $\mathbf{2}$ Left eye before treatment with sulfasalazine (use of fluorescein). Note: White arrows show temporal corneal thickening and infiltrate (staining with fluorescein) associated with adjacent limbal infiltrate.

A combination of topical (ciprofloxacin and indomethacin eye drops) and systemic treatment (oral ibuprofen) was started for 3 weeks, with no improvement on the clinical signs.

Diagnostic tests revealed an inflammatory syndrome and a CD4 count of $103 / \mu \mathrm{L}$. Systemic investigation was negative for syphilis, tuberculosis, and hepatitis B and C. Diagnostic workup of rheumatismal diseases included laboratory tests and biopsy of affected tissues. Testing for rheumatoid factor, Waaler-Rose reaction, antinuclear antibodies (ANA), anti-native DNA antibodies, antineutrophil cytoplasmic antibodies (ANCA), and anti-cyclic citrullinated peptide antibodies (anti-CCP) was negative. The assessment of the biopsied scleral nodule revealed an inflammatory lesion (vasculoexudative phase).

The patient also consulted a rheumatologist, and a diagnosis of RA was made based on the clinical findings according to American College of Rheumatology/European League 
Against Rheumatism (ACR/EULAR) classification criteria (patient's score $>6$ ).

Treatment with sulfasalazine $(500 \mathrm{mg} / \mathrm{d})$ was started, and was progressively increased (1,500 mg daily) over 3 weeks. Regression of symptoms, and ocular and articular signs was observed within 15 days (Figure 3 ).

The patient was monitored every month until November 2011. Sulfasalazine treatment was stopped in September 2011, and no recurrence of clinical signs was observed at the 8-month follow-up (best visual acuity of both eyes: 20/20). The clinical examination revealed an absence of scleritis in the right eye and a disappearance of intraocular inflammation associated to a temporal degeneration of the perilimbal conjunctiva in the left eye (Figures 4 and 5).

\section{Discussion}

In the literature, many authors have reported associations of multiple forms of chronic inflammatory rheumatism and HIV/AIDS disease in black African patients. ${ }^{1-10}$ However, the physiopathogenic mechanism is still unknown.

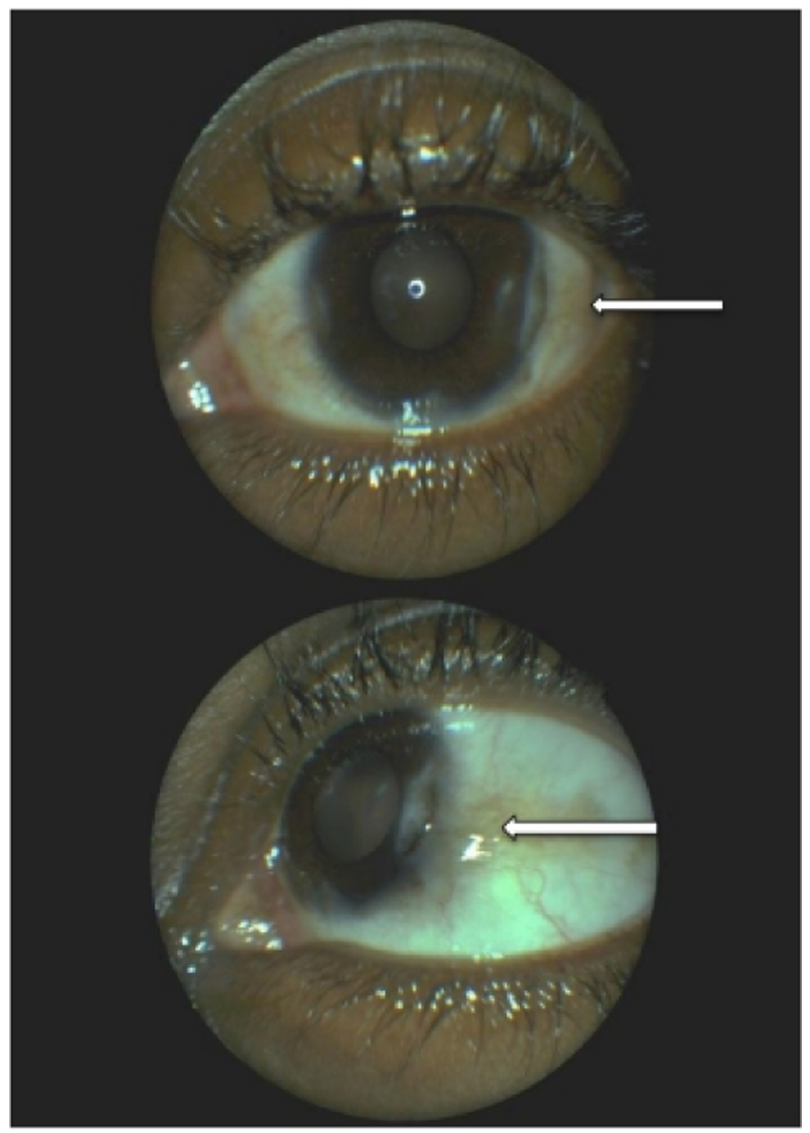

Figure 3 Left eye lesion I month after initiating the treatment with sulfasalazine. Notes: White arrows show persistence of the temporal thinning of the cornea associated with a mild degeneration of the temporal conjunctiva. Disappearance of the infiltrate around the limbus.

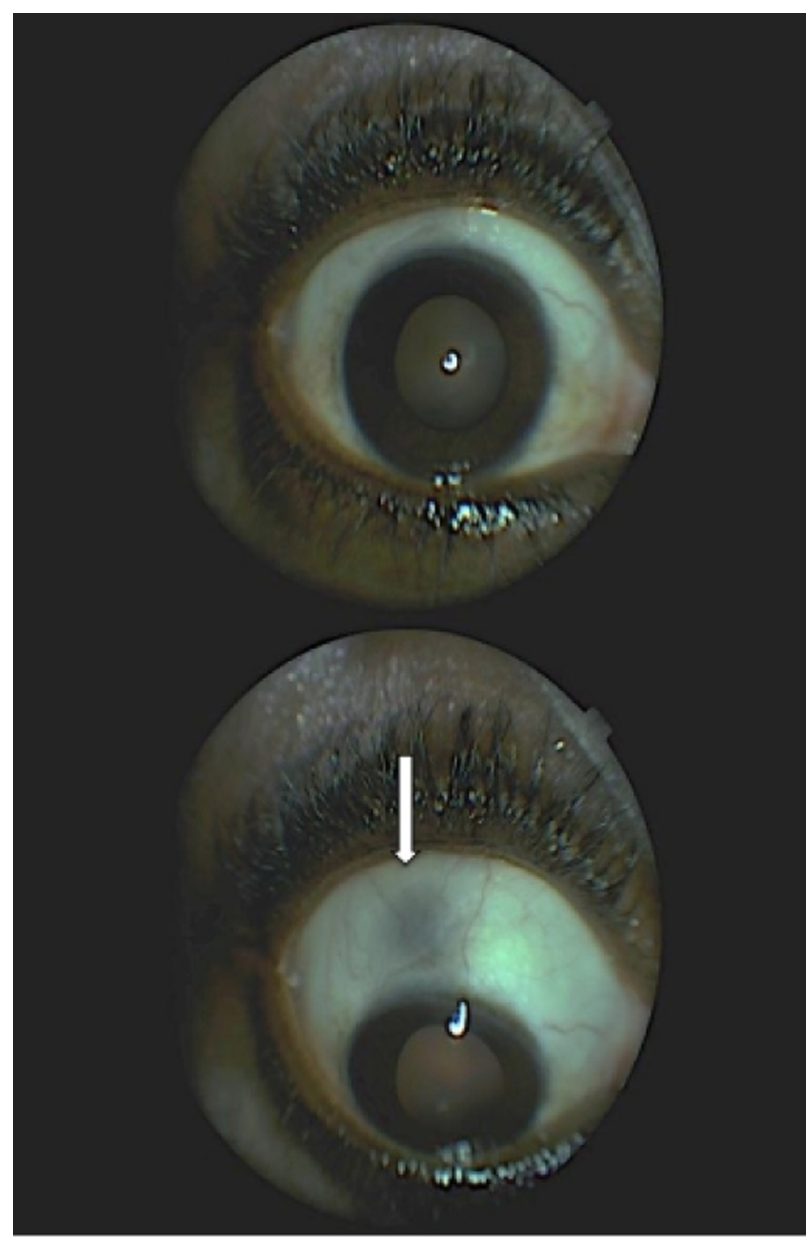

Figure 4 Right eye 8 months after initiating the treatment with sulfasalazine. Note: The white arrow shows no reoccurrence of the scleritis in the superior quadrant.

Ocular manifestations such as scleritis and peripheral ulcerative keratitis can be related to local or systemic infectious or to inflammatory causes. ${ }^{12}$ However, according to the literature, these types of ocular lesions are not specific to HIV/ AIDS infection and/or to antiretroviral treatment. Scleritis is an uncommon condition that predominantly affects females. The Watson and Hayreh classification of scleritis distinguishes two forms: anterior (diffuse, nodular, and necrotizing) and posterior forms. ${ }^{14}$ Much research, focused on the pathogenesis of scleritis, has suggested a link with inflammatory process mediated by Th cells. ${ }^{15}$ In the presence of anterior diffuse and nodular scleritis, systemic causes are found in 40\%-50\%, and infectious etiologies, in $7 \%$ of cases, ${ }^{16,17}$ whereas in necrotizing scleritis, systemic affections are found in 50\%-80\% of cases. ${ }^{16}$ In our case, the anterior nodular scleritis was an inflammatory process according to the pathologist (figure of the biopsied lesion not available).

RA and granulomatosis with polyangiitis are systemic diseases mainly associated with scleritis. ${ }^{16,18}$ 


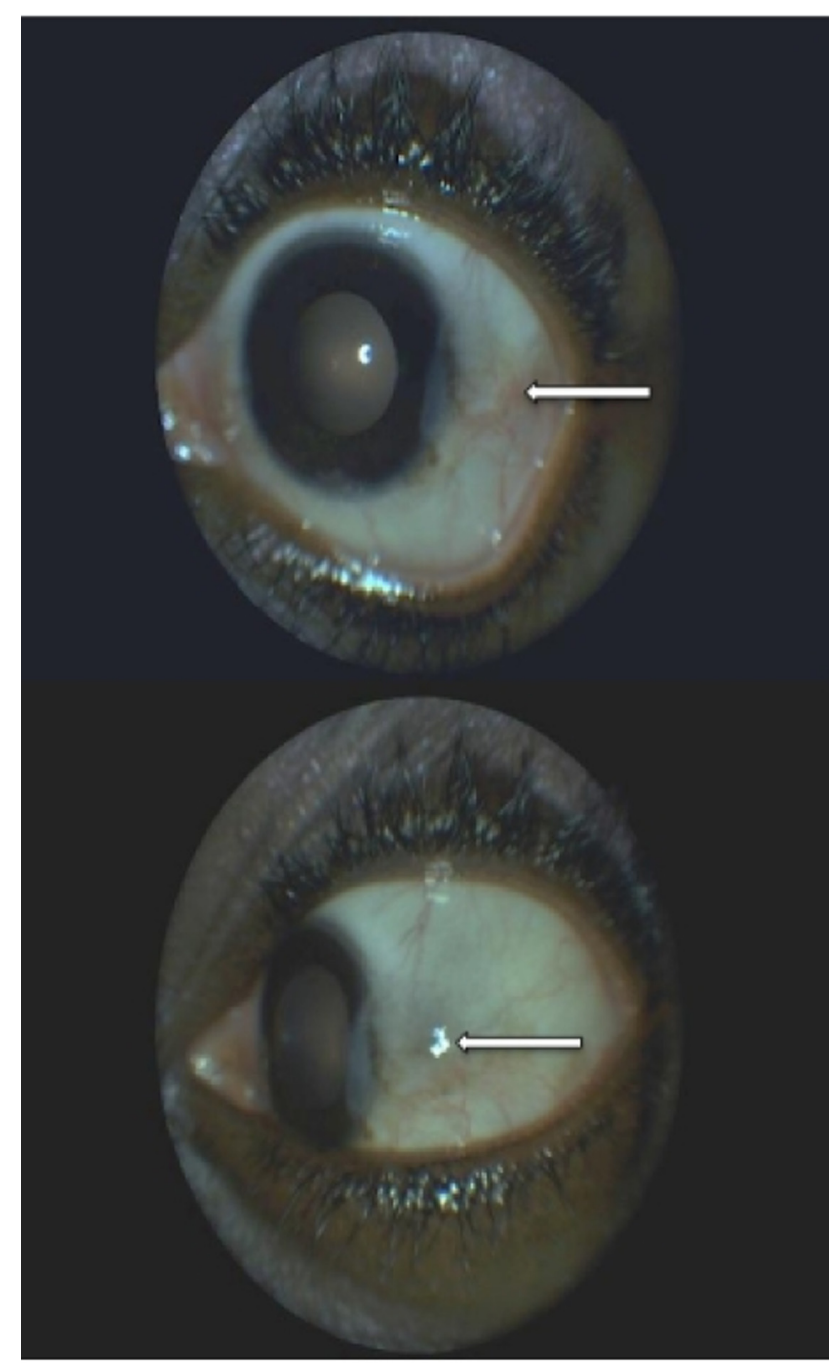

Figure 5 Left eye 8 months after initiating the treatment with sulfasalazine. Note: White arrows show persistence of the temporal degeneration of the conjunctiva around the limbus that is growing on the cornea.

Complications of anterior scleritis include anterior uveitis, peripheral ulcerative keratitis, cataract, or glaucoma. Sainz de la Maza et al, ${ }^{19}$ reported that patients with scleritis also had anterior uveitis, cataract, peripheral ulcerative keratitis, and glaucoma in $42 \%, 17 \%, 14 \%$, and $13 \%$ of cases, respectively. Peripheral ulcerative keratitis is an ocular disease that occurs less often than scleritis. It affects both sexes equally. Peripheral ulcerative keratitis is frequently associated with necrotizing scleritis and is bilateral in more than $40 \%$ of cases. ${ }^{20}$ Tauber et al, ${ }^{21}$ in their study, reported an association of ulcerative keratitis and RA in $34 \%$ of patients. In addition, corneal involvement was described as the first manifestation of RA.

According to Foster et al, ${ }^{22}$ mortality rate at 10 years in patients affected by RA associated with peripheral ulcerative keratitis and necrotizing scleritis could be estimated at $50 \%$.
The pathogenesis of peripheral ulcerative keratitis is still unknown. However, one hypothesis is that stromal destruction could be due to the collagenases and other proteases produced by the immune cells (neutrophils and macrophages). In addition, the presence of proinflammatory cytokines induces the release of metalloproteinases by stromal keratocytes, leading to the destruction of the cornea. ${ }^{23}$

Clinical, radiological, and biological investigations are similar in scleritis and peripheral ulcerative keratitis presentation. Tuberculosis, syphilis, and Lyme disease are the most frequent systemic infections related to these ocular manifestations, whereas RA and granulomatosis with polyangiitis appear to be the most common inflammations. ${ }^{12}$ According to the type of ocular lesions described in our case, the patient was not worked up for Sjögren syndrome (antiSSA and anti-SSB antibodies), which is known as a disease often associated to RA. ${ }^{24}$

In accordance with our patient's immunological status due to HIV infection, a workup of opportunistic infections in reference to ocular manifestations was first carried out, and all results were negative. Although the systemic investigation of rheumatismal affections was negative, the diagnosis of RA was retained in accordance to ACR/ EULAR classification criteria (2010). ${ }^{25}$ Moreover, good response to the trial treatment (sulfasalazine) was an additional contribution to this diagnosis. RA is described as an autoimmune disease characterized by chronic inflammation and articular destruction. It is the most frequent inflammatory arthritis found in females. Predisposing genetic factors of RA have been reported, although its pathophysiologic mechanism is poorly understood human leukocyte antigen (HLA)-DR. Moreover, inflammation in RA is characterized by high erythrocyte sedimentation rate and/ or C-reactive protein, the presence of rheumatoid factors, and more specifically, a high level of anti-CCP, although the diagnosis of RA, is primarily based on clinical features (existence of seronegative forms). ${ }^{26,27}$ However, according to the literature, the association of RA with HIV infection is quite rare. ${ }^{11}$ Moreover, HIV infection may lead to antiCCP antibody positivity, and RA to false-positive HIV serology. In our case, the search for anti-CCP antibodies was negative, and the diagnosis of HIV was made few years earlier to the actual clinical presentation.

Therapy for RA combines the use of anti-inflammatory and immunosuppressive drugs. Local and systemic anti-inflammatory drugs essentially act on the acute phase by stopping the inflammation (nonsteroidal anti-inflammatory, corticosteroids). Systemic disease modifying antirheumatic 
drugs (DMARDs), ${ }^{28}$ more often combined to anti-inflammatory ones, slow down, or even stop the clinical course of the disease. There are two types of DMARDs, conventional ones (cyclosporine, cyclophosphamide, gold injections, hydroxychloroquine, leflunomide, methotrexate, mycophenolate, sulfasalazine) and biological therapies (anti-TNF [tumour necrosis factor] drugs). DMARDs should be started as soon as the diagnosis of RA is made. ${ }^{29}$ Many authors evoke the complexity of therapeutic management in chronic inflammatory rheumatisms due to numerous side effects and potential complications (myelosuppression, renal and liver toxicity, skin rash, gastrointestinal disturbance, infections). ${ }^{10,28}$ Thus, therapy with DMARDs should be initiated by rheumatologists. Moreover, patients treated with these drugs should be monitored regularly, and any complications seen should be the motive for stopping the treatment. ${ }^{28}$

In this case, the treatment choice of the rheumatologist was sulfasalazine, and a favorable response was obtained within few weeks. Sulfasalazine is a well-known treatment for RA. Its efficacy has been demonstrated in several clinical trials. ${ }^{30}$ It acts by inhibiting arachidonic acid synthesis (anti-inflammatory action), and it also has an immunosuppressive action with a poorly understood mechanism. It is well tolerated and remains the first line treatment in many countries. Its main contraindication is salicylate hypersensitivity. It can be used alone or in association with immunosuppressive drugs. ${ }^{29,30}$ Although, there is little evidence to recommend the use of DMARDs when RA coexists with HIV/AIDS infection, ${ }^{11}$ sulfasalazine was well-tolerated by our patient with a complete recovery of ocular lesions (duration of treatment: 6 months).

\section{Conclusion}

We have described the clinical course of ocular manifestations (nodular scleritis, peripheral ulcerative keratitis, and anterior uveitis) in the context of polyarthropathies in a HIV-positive female. In this case report, RA diagnosis was based on ACR/EULAR classification criteria. Coexisting pathologies should always be considered, and diagnosis of chronic inflammatory rheumatism workup combined to a complete ocular examination should be made, even in the context of HIV disease.

\section{Author contributions}

All authors contributed toward data analysis, drafting and revising the paper and agree to be accountable for all aspects of the work.

\section{Disclosure}

The authors report no conflicts of interest in this work.

\section{References}

1. Stein M, Davis P. HIV and arthritis - causal or casual acquaintances? J Rheumatol. 1989;16(10):1287-1290.

2. Bileckot R, Mouaya A, Makuwa M. Prevalence and clinical presentations of arthritis in HIV-positive patients seen at a rheumatology department in Congo-Brazzaville. Rev Rhum Eng Ed. 1998;65(10):549-554.

3. Njobvu P, McGill P, Kerr H, Jellis J, Pobee J. Spondyloarthropathy and human immunodeficiency virus infection in Zambia. J Rheumatol. 1998;25(8):1553-1559.

4. Stein CM, Davis P. Arthritis associated with HIV infection in Zimbabwe. J Rheumatol. 1996;23(3):506-511.

5. Njobvu P, McGill P. Human immunodeficiency virus related reactive arthritis in Zambia. J Rheumatol. 2005;32(7):1299-1304.

6. Chinniah K, Mody GM, Bhimma R, Adhikari M. Arthritis in association with human immunodeficiency virus infection in Black African children: causal or coincidental? Rheumatology (Oxford). 2005;44:915-920.

7. Njobvu P, McGill P. Soft tissue rheumatic lesions and HIV infection in Zambians. J Rheumatol. 2006;33(12):2493-2497.

8. Ekwom PE, Oyoo GO, Amayo EO, Murilithi IM. Prevalence and characteristics of articular manifestations in human immunodeficiency virus infection. East Afr Med J. 2010;87(10):408-414.

9. Ntsiba H, Ngandeu-Singwé M, Makita-Bagamboula C, Yala F. Les arthrites liées au virus de l'immunodéficience humaine au Congo Brazzaville [Human immunodeficiency virus associated arthritis in Congo Brazzaville]. Médecine et Maladies Infectieuses. 2007;37(11):758-761. French.

10. Tikly M, Njobvu P, McGill P. Spondyloarthritis in sub-Saharan Africa. Curr Rheumatol Rep. 2014;16:421.

11. Cunha BM, Mota LM, Pileggi GS, Safe IP, Lacerda MV. HIV/AIDS and rheumatoid arthritis. Autoimmun Rev. 2015;14(5):396-400.

12. Galor A, Thorne JE. Scleritis and peripheral ulcerative keratitis. Rheum Dis Clin North Am. 2007;33(4):835-854.

13. Govender P, Hansraj R, Naidoo KS, Visser L. Ocular manifestations of HIV/AIDS: a literature review. S Afr Optom. 2011;70(2):81-88.

14. Watson PG, Hayreh SS. Scleritis and episcleritis. Br J Ophthalmol. 1976;60(3):163-191.

15. Fong LP, Sainz de la Maza M, Rice BA, Kupferman AE, Foster CS Immunopathology of scleritis. Ophthalmology. 1991;98(4):472-479.

16. Schwam B. Scleritis. In: Krachmer J, Mannis M, Holland E, editors. Cornea and External Disease: Clinical Diagnosis and Management. Vol 2 St Louis, MO: Mosby; 1997:1479-1491.

17. Akpek EK, Thorne JE, Qazi FA, Do DV, Jabs DA. Evaluation of patients with scleritis for systemic disease. Ophthalmology. 2004;111(3):501-506.

18. Tuft SJ, Watson PG. Progression of scleral disease. Ophthalmology. 1991;98(4):467-471.

19. Sainz de la Maza M, Jabbur NS, Foster CS. Severity of scleritis and episcleritis. Ophthalmology. 1994;101(2):389-396.

20. Sainz de la Maza M, Foster CS, Jabbur NS, Baltatzis S. Ocular characteristics and disease associations in scleritis-associated peripheral keratopathy. Arch Ophthalmol. 2002;120(1):15-19.

21. Tauber J, Sainz de la Maza M, Hoang-Xuan T, Foster CS. An analysis of therapeutic decision making regarding immunosuppressive chemotherapy for peripheral ulcerative keratitis. Cornea. 1990;9(1):66-73.

22. Foster CS, Forstot SL, Wilson LA. Mortality rate in rheumatoid arthritis patients developing necrotizing scleritis or peripheral ulcerative keratitis: effects of systemic immune suppression. Ophthalmology. 1984;91: $1253-1263$.

23. Dana M, Qian Y, Hamrah P. Twenty-five-year panorama of corneal immunology: emerging concepts in the immunopathogenesis of microbial keratitis, peripheral ulcerative keratitis, and corneal transplant rejection. Cornea. 2000;19(5):625-643. 
24. He J, Ding Y, Feng M, et al. Characteristics of Sjogren syndrome in rheumatoid arthritis. Rheumatology (Oxford). 2013;52(6):1084-1089.

25. Aletaha D, Neogi T, Silman AJ, et al. 2010 Rheumatoid arthritis classification criteria: an American College of Rheumatology/European League Against Rheumatism collaborative initiative. Arthritis Rheum. 2010;62(9):2569-2581.

26. Rozin AP, Hasin T, Toledano K, Guralnik L, Balbir-Gurman A. Seronegative polyarthritis as severe systemic disease. Neth $\mathrm{J} \mathrm{Med}$. 2010;68(6):236-241.

27. Ouédraogo DD, Singbo J, Diallo O, Sawadogo SA, Tiéno H, Drabo YJ. Rheumatoid arthritis in Burkina Faso: clinical and serological profiles. Clin Rheumatol. 2011;30(12):1617-1621.
28. Chakravarty K, McDonald H, Pullar T, et al. BSR/BHPR guideline for disease-modifying anti-rheumatic drug (DMARD) therapy in consultation with the British Association of Dermatologists. Rheumatology (Oxford). 2008;47(6):1-16.

29. Smolen JS, Landewé R, Breedveld FC, et al. EULAR recommendations for the management of rheumatoid arthritis with synthetic and biological disease modifying anti rheumatic drugs: 2013 update. Ann Rheum Dis. doi: 10.1136/annrheumdis-2013-204573.

30. Plosker GL, Croom KF. Sulfasalazine: a review of its use in the management of rheumatoid arthritis. Drugs. 2005;65(13):1825-1849.

\section{Publish your work in this journal}

The International Medical Case Reports Journal is an international, peer-reviewed open-access journal publishing original case reports from all medical specialties. Previously unpublished medical posters are also accepted relating to any area of clinical or preclinical science. Submissions should not normally exceed 2,000 words or
4 published pages including figures, diagrams and references. The manuscript management system is completely online and includes a very quick and fair peer-review system, which is all easy to use. Visit http://www.dovepress.com/testimonials.php to read real quotes from published authors.

Submit your manuscript here: http://www.dovepress.com/international-medical-case-reports-journal-journal 